\title{
Lazer Tabanlı Dijital Ölçüm Sistemi ile Piezoelektrik Eyleyicilerin Rezonans ve Anti-Rezonans Frekanslarının Belirlenmesi
}

\author{
Levent Paral1 ${ }^{*}$, Özge Cihanbeğendi Şahin ${ }^{2}$, Ali Sarı ${ }^{3}$, Jiri Pechousek ${ }^{4}$ \\ ${ }^{1 *}$ Celal Bayar Üniversitesi, Turgutlu Meslek Yüksekokulu, Elektronik ve Otomasyon Bölümü \\ Turgutlu, Manisa, +90 2363145508 \\ levent.parali@cbu.edu.tr \\ 2Dokuz Eylül Üniversitesi Mühendislik Fakültesi Elektrik Elektronik Mühendisliği Bölümü \\ Buca, İzmir, +90 2323017179 \\ ozge.sahin@deu.edu.tr \\ ${ }^{3}$ Celal Bayar Üniversitesi, Turgutlu Meslek Yüksekokulu, Elektrik ve Enerji Bölümü \\ Turgutlu, Manisa, +90 2363145508 \\ ali.sari@cbu.edu.tr \\ ${ }^{4}$ Palacky University, Department of Experimental Physics, Faculty of Science, \\ Olomouc, Czech Republic, +420 585634949 \\ jiri.pechousek@upol.cz \\ *Iletişimden sorumlu yazar/Correspongin author \\ Geliş/Recieved: 12 Aralık (December) 2016 \\ Kabul/Accepted: 2 Mayis (May) 2017 \\ DOI: 10.18466/cbayarfbe.319957
}

\begin{abstract}
Özet
Günümüzde piezoelektrik sensörler, piezoelektrik patchler aracılığıyla çelik yapıların sağlamlık kontrollerinde, mekanik enerjinin elektrik enerjiye çevrilmesiyle güç hasat toplama üniteleri için gerekli elektrik depolamalama sistemlerinde, uçak gövde yapılarının izlenmesinde ve mekatronik sistemlerin hareketli aksamları gibi birçok alanda kullanılmıştır. Aynı zamanda piezoelektrik sensörler, buzzer olarak ses simülatörlerinde, mikrofonlarda, kulaklıklarda, basınç ölçümlerinde, sualtındaki kablosuz ultrasonik haberleşme sistemlerinde ve medikal ekipmanlarında da kullanılmıştır. Bu çalışma, bir titreşim-ölçer (vibrometre) olarak lazer yer değiştirme sensör tabanlı dijital ölçüm sisteminin yenilikçi bir tasarımını tanıtır. Aynı zamanda lazer yer değiştirme sensör tabanlı dijital bir ölçüm sistemi tarafından uyartılan bir piezoelektrik eyleyicinin titreşim özelliklerine göre, rezonans -antirezonans frekans özelliklerini belirlemeyi amaçlar. Piezoelektrik eyleyicinin titreşim tepkimesi, işaret üreticinin çıkışı ve sayısallaştırıcı kart girişinin bir kişisel bilgisayar tarafından işlenmesiyle elde edilmiştir. Titreşimin rezonans frekansı, lazer yer değiştirme sensörü tarafından ölçülen sinyal tepkimesindeki süpürmeli sinusiodal uyartımlarını izleyen pik değerlerinden faydalanılarak ölçülmüştür. Maksimum yer değiştirmeye sahip rezonans frekans sonucu, büyük ölçüde piezoelektrik diskin iyi sabitlenmesine bağlıdır. Elde edilen ölçüm sonuçları, kurulunan dijital ölçüm sisteminin, piezoelektrik diskin rezonans frekanslarını 20 MHz'e kadar 1 Hz'lik çözünürlükte ölçerek ayırt edebilir kapasitede olduğunu gösterir. Lazer yer değiştirme sensörünün kontrol ünitesinden elde edilen analog sinyal, piezoelektrik eyleyicinin mekaniksel titreşimiyle doğrudan ilişkilidir. Piezoelektrik eyleyicinin elektromekanik kublaj faktörü-k, rezonans ve antirezonans frekans değişimlerine göre hesaplanmıştır. Elde edilen sonuçlar, piezoelektrik eyleyici üretici firmasının deklare ettiği katolog değerleriyle uyumludur.
\end{abstract}

Anahtar Kelimeler -Dijital ölçüm sistemi, lazer yer değiştirme sensörü, rezonans-antirezonans frekansları 


\title{
Determining Resonance and Antiresonance Frequencies of Piezoelectric Actuator by Digital Measurement System Based on Laser
}

\begin{abstract}
Nowadays, piezoelectric sensors are used in many areas such as health monitoring of steel structures using thickness modes study of piezoelectric patches, in electricity storage systems for power harvesting by converting a mechanical energy into an electrical one, in monitoring a body structure of aircrafts, in the moving parts of the mechatronics systems. The piezoelectric sensors also are used in such voice stimulators as a buzzer, microphones, head phones, for pressure measurement, in the underwater wireless ultrasonic communication, and in the medical equipment. This study describes the innovative design of a digital measurement system based on a laser displacement sensor as a vibrometer and it also aims to be capable of determning the resonance-antiresonance frequency properties of a piezoelectric actuator excited by a digital measurement system based on laser displacement sensor. The vibration response of the piezoelectric actuator is obtained by processing the input/output signals achieved from the function generator and digital oscilloscope cards driven by a personal computer. Resonant frequencies of vibration are achieved utilizing the sweep-sine signal excitation following the peak values in the signal response measured by laser displacement sensor. The results of the resonance frequency together with the maximum displacement measurements highly depend on the fixation of the piezoelectric disc. The test measurement results show that the system can distinguish resonance frequencies of pizeoelectric discs up to $20 \mathrm{MHz}$. with the resolution $1 \mathrm{~Hz}$. The analogue signal from laser displacement sensor's controller associated with directly a mechanical vibration of a piezoelectric actuator. The electromechanical coupling coefficent-k of the piezoelectric actuator is calculated by using resonance-antiresonance frequencies. The results achived are in compliance with the reference values proclaimed by the manufacturer of the piezoelectric actuator.
\end{abstract}

Keywords - Digital measurement system, laser displacement sensor, resonance-antiresonance frequencies,

\section{Giriş}

Piezoelektrik materyaller, doğasında bulunan dönüştürücü özelliklerinden dolayı akıllı yapılar olarak çok yaygın kullanılmaktadır. Piezoelektrik metaryallerde mekaniksel yüklemeden veya titreşimden dolayı boyutsal bir değişim meydana geliyorsa, onlar alg1lama (sensör) özelliklerinden dolayı elektrik gerilimi üretirler. Eğer bir piezoelektrik materyal eyleyici (aktuatör) olarak kullanılıyorsa, onlar kendisine uygulanan alternatif gerilimin frekansına eşit bir frekans değerinde titreşim sergilerler. [1, 2]

Piezoelektrik eyleyiciler bir uyarıcı olarak kullanıldığında, onların uygulanan gerilimine bağlı yer değiştirme yeteneklerinden dolayı, atomik kuvvet mikroskopları [3], hızlandırıcı tabanlı görüntü sistemleri [4], bilgisayar ekipmanları [5] , makine ve endüstriyel robot uygulamaları $[6,7]$ gibi çok çeşitli uygulama alanlarında tercih edilirler. Aynı zamanda piezoelektrik algılayıcı olarak mikrofonlarda, basınç ölçümlerinde, sualtı kablosuz ultrasonik haberleşme sis- temlerinde ve medikal cihazlarında da kullanılırlar. Titreşen bir yüzeyin mekaniksel olarak yer değişimi, denge şartlarında sıfır konumundaki pozisyonundan yukarı-aşağı yönde uzaklaşması olarak tanımlanır. Genellikle titreşim ölçümleri, verilen frekans aralığında, ayrı ayrı veya eş zamanlı hız, ivme ve yer değiştirme miktarları olarak karakterize edilebilir. Lazer tabanlı yer değiştirme sensörleri (Laser Displacement Sensor), titreşen bir yüzeyin titreşim miktarlarını yer değişim miktarı olarak temassız optik bir yöntemle gerçekleştirme yeteneğine sahiptir. $[8,9$, 10]

Bir piezoelektrik materyalin elektrik enerjisini mekanik enerjiye veya bir mekanik enerjisini elektrik enerjisine dönüştürme verimi, elektromekanik kublaj faktörü-k ile ifade edilir. Piezoelektrik eyleyicilerin, kendisine uygulanan elektrik enerjisini titreşerek mekanik enerjisine dönüştürme yeteneği de elektromekanik kublaj faktörü-k ile tanımlanır. 
Bir piezoelektrik eyleyici, en yüksek genlikteki (en düşük empedansta) rezonans frekansı (fr) ile en düşük genlikli (en yüksek empedansta) antirezonans frekansı(fa) aralığında titreşim sergiler.[11] Böylece, bir piezoelektrik eyleyicinin rezonans ve antirezonans çalışma frekanslarının belirlenmesiyle, aşağıdaki denklem yardımıyla elektromekanik kublaj faktörü-k hesaplanabilir.

$$
k^{2}=\frac{\left(\mathrm{fa}^{2}-\mathrm{fr}^{2}\right)}{\mathrm{fa}^{2}}
$$

Burada, k elektromekanik kublaj faktörünü, fr rezonans frekansını, fa ise antirezonans frekansını ifade eder. [11]

Bu çalışmada, lazer tabanlı dijital bir ölçüm sistemi oluşturulmuş, bir piezoelektrik eyleyicinin rezonans ve antirezonans frekanslarının ölçülmesi ve ölçülen değerlere göre elektromekanik kublaj faktörünün belirlenmesi amaçlanmıştır. Lazer yer değiştirme sensörü, bir ışı̆̆a duyarlı bir aygit (CCD: Charge Coupled Device) üzerindeki lazer ışığının yansımasını kullanarak çalışır. Geliştirilen dijital ölçüm sistemiyle, test edilen piezoelektrik eyleyicinin maksimum titreşim yer değiştirmesi ölçülmüştür.

\section{Materyal ve Metot}

$\mathrm{Bu}$ çalışmada, Murata firmasının ses komponenti olarak bilinen 7BB 27-4 tanımlı piezoelektrik eyleyici kullanılmıştır. Piezoelektrik eyleyici-disk'in diyafram yapısı, pirinç bir materyal üzerine PZT (kurşun titanyum zirkonyum oksit: Piezo Seramik) eklenmesiyle üretilmiştir (Şekil.1) (Çizelge 1).
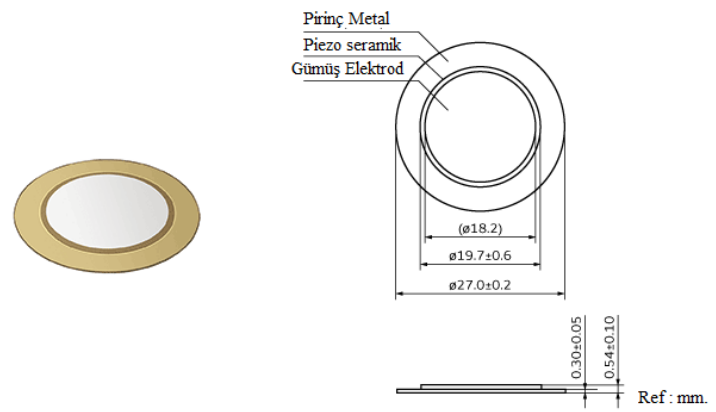

Şekil 1. Piezoelektrik eyleyici - disk (7BB 27-4)
Çizelge 1. Piezoelektrik Eyleyici-Diskin Özellikleri.

\begin{tabular}{ll} 
Model No & 7BB-27-4 \\
\hline Element tipi & Piezo \\
Yapısı & Lead Titan. Zirc. Ox. \\
Dönüştürücü Fonksiyonu & Diyafram \\
Plate Çapı, D (mm) & 27.0 \\
Element Çapı, a (mm) & 19.7 \\
Electrod Çapı, b (mm) & 18.2 \\
Kalınlık, T (mm) & 0.54 \\
Metal Plate Kalınlığı, tm (mm) & 0.30 \\
Rezonans Frekansı, fr (kHz) & $4.6 \pm 0.5$ \\
Kapasitesi, C (nf) & $20 \pm 30 \%$ \\
Rezonans Empedansı, R (ohm) & $\leq 200$ \\
\hline
\end{tabular}

Kurulan lazer tabanlı dijital ölçüm sistemi, LK-G3001 kontrol ünitesi tarafından kontrol edilen LK-G37 keyence lazer yer değiştirme sensörü (Şekil.2), PCI5402 sinyal üretici (National Instruments), PCI-5122 dijital osilaskop (National Instruments), güç yükselteci ve bir kişisel bir bilgisayardan meydana gelmektedir.
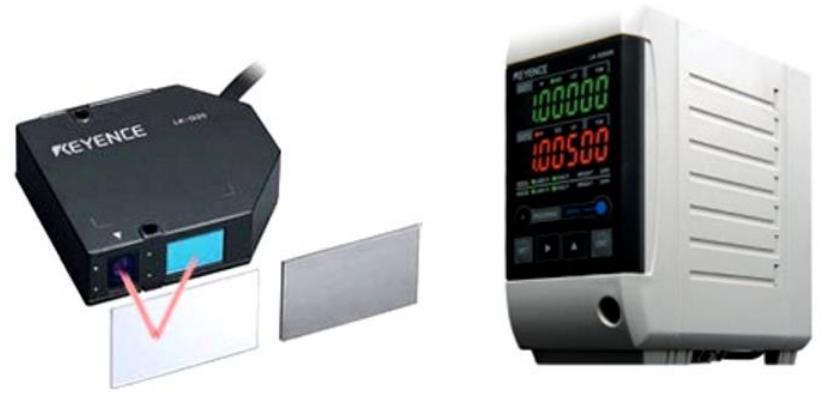

Şekil 2. Keyence lazer yer değiştirme sensörü ve kontrol ünitesi

Sistemin ana konsepti, LabVIEW ${ }^{\mathrm{TM}}$ de kullanilan sanal enstrüman tekniği temeline dayanmaktadır. Ölçüm sisteminin deneysel kurulumu Şekil.3 'de, piezoelektrik eyleyicinin ölçüm düzeneğine sabitlenmesi ve merkezine odaklanmış lazer ışınının gerçek görüntüsü de Şekil.4'de gösterilmiştir.

Kontrol sistemi iki adet PCI tabanlı data toplama kartlı bir kişisel bilgisayardan meydana gelmiştir. PCI 5402 sinyal üretici aracılığıyla belirli bir frekans aralığında ve uyartım geriliminde uyartılan piezoelektrik eyleyici-disk titreşmeye başlar. 


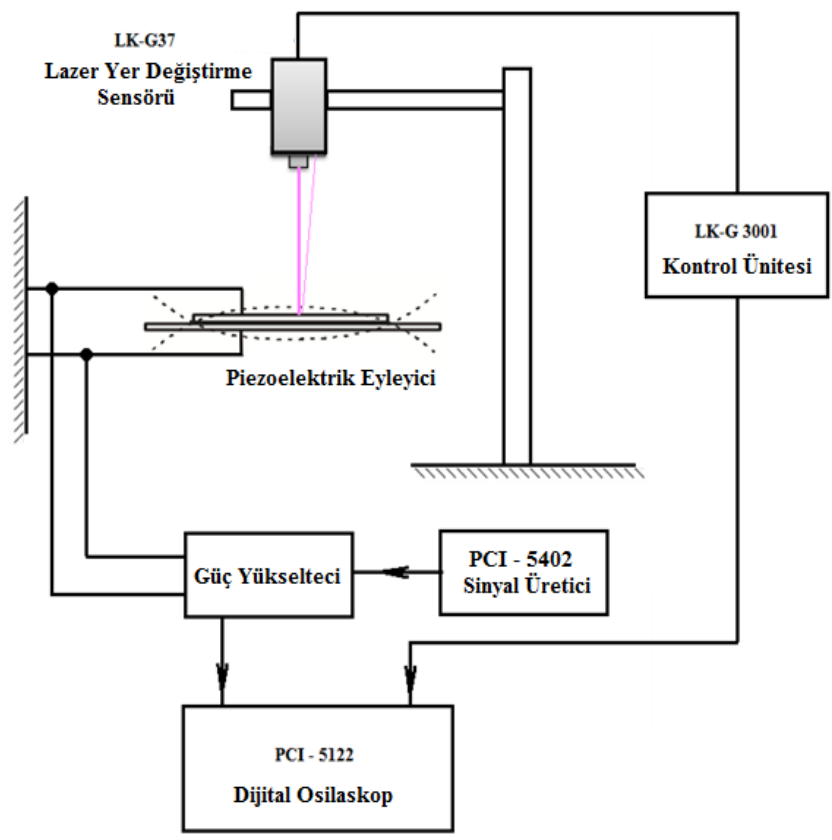

Şekil 3. Lazer yer değiştirme sensör tabanlı dijital ölçüm sistemi.

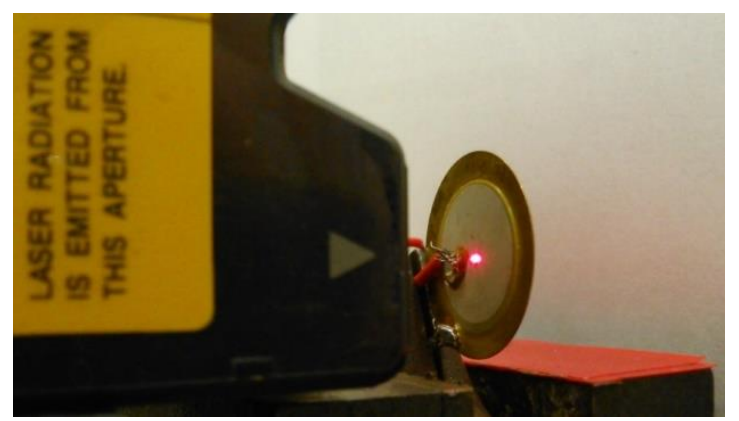

Şekil 4. Lazer ışığının piezoelektrik eyleyici merkezine odaklandırılması.[12]

Piezoelektrik eyleyici-diskin yüzeysel titreşimleri, lazer yer değiştirme sensörü aracilığıyla temassız olarak, yer değişim miktarı cinsinden algılanır. Algılanan 0-10 V' luk titreşimin analog verileri, PCI 5122 sayısallaştırıcı kart üzerinden dijital verilere çevrilerek, özel geliştirilmiş LabVIEW ${ }^{\mathrm{TM}}$ tabanlı yazılım algoritmalar aracılığıyla yüksek örnekleme aralıklarında yüksek çözünürlüğe sahip veriler elde edilir. Sistem, sinüs sinyal üreticiyle uyartılan bir piezoelektrik eyleyicinin titreşim tepkimesine bağlı olarak dinamik yer değiştirme miktarını ölçebilecek kapasitedir. [12]

\section{Kullanıcı Arayüzü Parametre Ayarları}

Ölçüm sisteminin kontrol uygulaması için özel geliş- tirilmiş LabVIEWTM tabanlı kullanıcı arayüzü Şekil.5 'de gösterilmiştir.

Ölçüm sistemine ait kullanıcı arayüzü, ölçüm ve başlatma menüsü ayarları ile ölçüm sisteminden toplanan sinyallerin grafiksel olarak izlenebildiği kısımlardan meydana gelmektedir. Ölçüm sistemine ait ayarlar, aşağıda belirtilen işlem basamaklarına göre yapılır.

\section{1 Ölçüm Ayarları}

- Başlangıç Frekansı (Start Frequency): Tarama yapılacak frekans aralığının başlangıç frekansınin tanımlanması.

- Frekans Adımı (Frequency Step): Tarama frekansının kaç $\mathrm{Hz}$ adımlar ile taranacağının belirlenmesi.

- Bitiş Frekansı (Stop Frequency): Tarama yapılacak frekans aralığının bitiş frekansının tanımlanması.

- Nokta Sayısı (Number of points): Belirlenen frekans aralığındaki alınan ölçüm sayısının belirlenmesi. ( $\mathrm{Bu}$ değerin artması ile belirlenen frekans aralığındaki ölçüm sayısı daha az olacağından, hassasiyet azalacaktır)

- Ölçüm Tipi (Type of Meas): Hassas ve kaba ölçümler için seçim butonu.

- Hızlı Sekme Ayarı (Fast Elements): Hizlı sekme aracılığıyla ölçüm yapılacak gerilim sayının belirlenmesi.

- Uyartım Voltajı (Excitation Voltage): Uyartım gerilim aralıklarının belirlenmesi.

\subsection{Başlatma Menüsü Ayarları}

- Hassaslık Aralığı (Fine Elements): Ölçüm aralık sayısınin belirlenmesi.

- Örnekleme Oranı (Sample Rate): Lazer yer değiştirme sensörünün hassasiyetine bağlı olarak değişim gösteren örnekleme oranıdır. Kullandığımız LK-G37 lazer yer değiştirme sensörü için $2 \mathrm{MS} / \mathrm{s}$ oranı seçilmiştir.

- Periyod Sayisı (Number of Periods): Kullandığımız LK-G37 lazer yer değiştirme sensörü için 20 değeri seçilmiştir.

- Başlatma/Durdurma (Start/Stop): Ölçüm işlemini başlatılmasını veya durdurulmasını sağlayan butonlardir. 


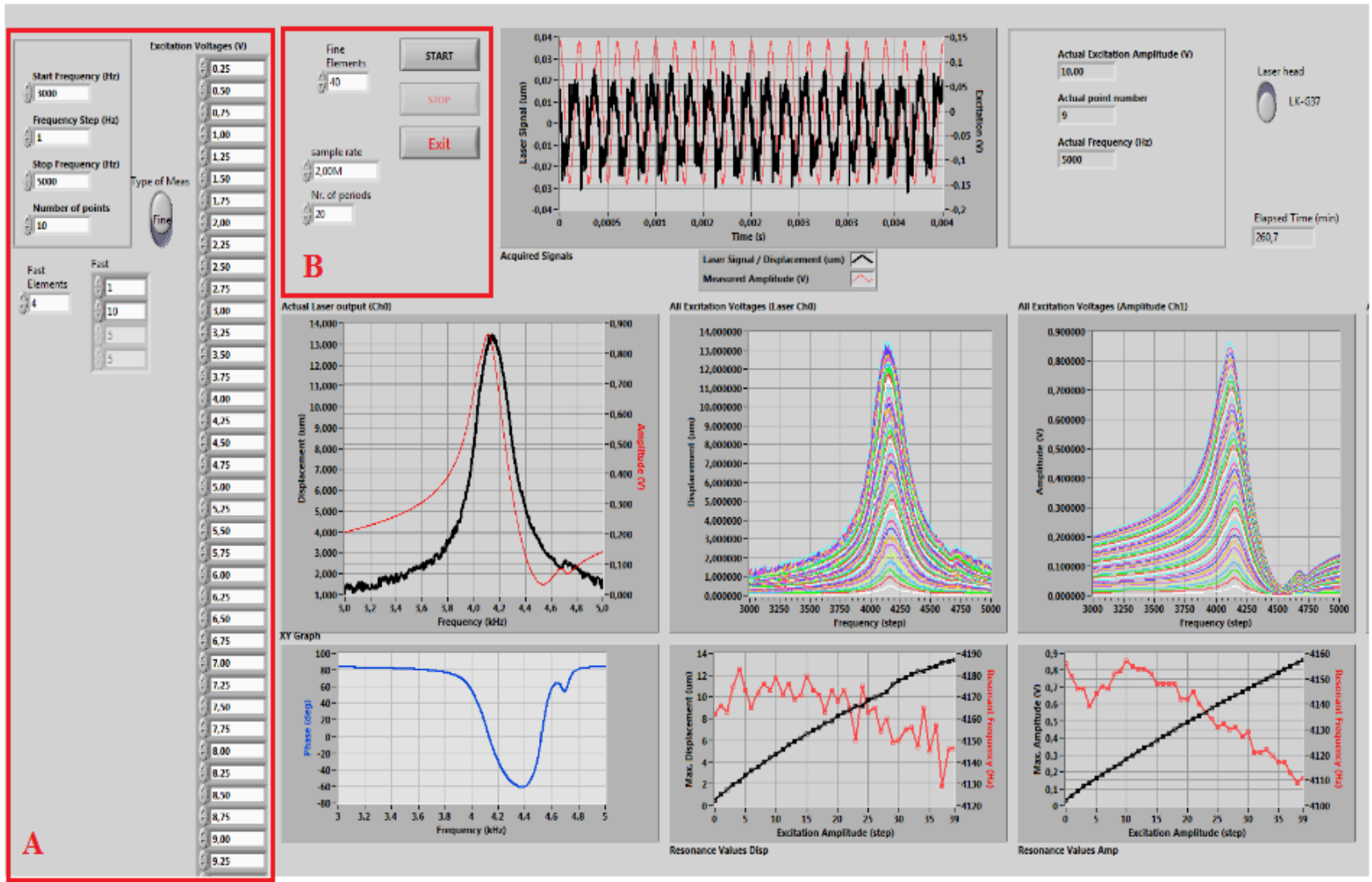

Şekil 5. Dijital ölçüm sisteminin LabVIEWTM tabanlı kullanıcı ara yüz görüntüsü.

\section{Bulgular ve Tartışma}

Ara yüzde yer alan kontroller, ölçümden önce ölçüm sisteminin ard arda çalışma davranış parametrelerinin, kullanıcı tarafından belirlenebilmesi için tasarlanmıştır. Ara yüzde bulunan göstergeler, lazer yer değiştirme sensöründen elde edilen analog değerleri temsil eden sayısallaştırıcı giriş sinyallerinin gerçek frekans ve genlik değerlerini gösterir. Ara yüzde bulunan grafiklerde, piezoelektrik eyleyici-diskin uyartım gerilimine bağlı olarak yer değiştirme ve frekans değişimleri gösterilmektedir.

Titreşimin rezonans frekansı, lazer yer değiştirme sensörü tarafından ölçülen frekans tepkimesinin tepe değeri olarak tanımlanır. Uyartım gerilimindeki rezonans frekansı ve maksimum yer değiştirme miktarı, ayrı ayrı grafiklerde gösterilmiştir. Ara yüz tarafından ölçüm süresince toplanan ölçüm sonuçları, excel formatında bilgisayar ortamına kayıt edilmiştir.

Bu çalışmada analizlenen piezoelektrik eyleyici diskin yer değiştirme özellikleri, lazer yer değiştirme sensör tabanlı ölçüm sistemi tarafından belirlenmiştir.

Uyartım gerilimi altındaki frekansa bağlı titreşimin yer değiştirme değerleri, Şekil.6 'da gösterilmiştir. Ölçümün frekans taraması, $1 \mathrm{~Hz}$ 'lik sinüs sinyal adımlarından oluşan $1-10 \mathrm{kHz}$ aralığını kapsamaktadir.

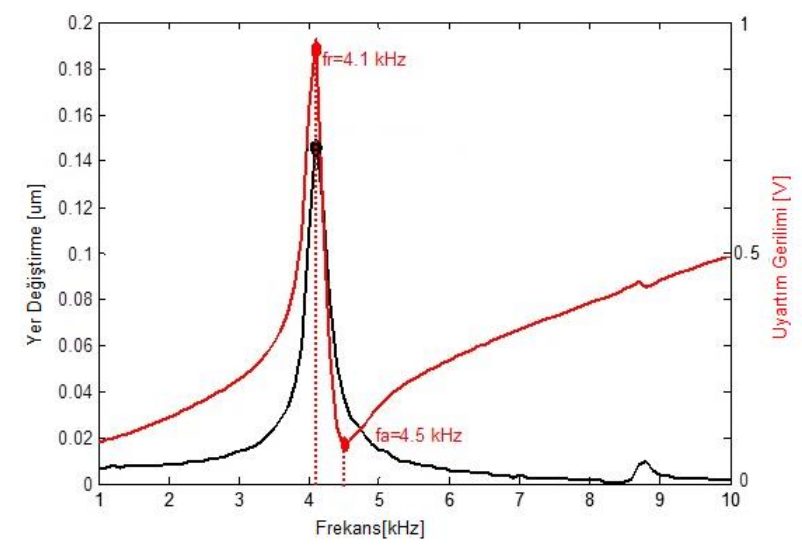

Şekil 6. Piezoelektrik eyleyicinin rezonans antirezonans frekanslarındaki yer değişimleri. 
Şekil.6 'daki kırmızı eğride görüleceği gibi, piezoelektrik eyleyici diskin rezonans frekansı (fr), maksimum yer değiştirmenin tepe değerindeki 4,1 kHz.'de, antirezonans frekansı ( $\mathrm{fa}$ ) da yer değiştirmenin en az olduğu 4,5 kHz. 'de meydana gelmiştir. Grafikteki siyah renkli eğri ise piezoelektrik eyleyici diskin sadece rezonans frekans tepkimesini göstermektedir.

\section{Sonuç}

$\mathrm{Bu}$ çalışmada, piezoelektrik eyleyicilerin titreşim karakterizasyonuna göre rezonans-antirezonans frekanslarının tespit edilmesi için lazer yer değiştirme sensör tabanlı dijital bir ölçüm sistemi açıklanmıştır. Ölçüm sistemi, ölçüm sistemine sabitlenmiş piezoelektrik eyleyici diskin, sinüs uyartım sinyaline bağlı olarak titreşim tepkimesini ve dinamik yer değiştirme danimiklerini ölçebilmek için geliştirilmiştir. Yer değiştirme eğrilerinden elde edilen rezonans ve antirezonans frekansları sırasıyla $4,1 \mathrm{kHz}$ ile $4,5 \mathrm{kHz}$ 'dir. Elde edilen rezonans ve antirezonans frekans değerlerine göre elektromekanik kublaj faktörü-k 0,41 (dönüştürme verimi: \%41) olarak hesaplanmıştır. Kurulan dijital ölçüm sistemi, sadece piezoelektrik eyleyicilerin titreşim karakteristiklerinin tanımlanması için değil aynı zamanda plastik-metal metaryeller, transparan filmler ve hard disk gibi ekipmanların konumlandırılması ve yüzey özelliklerinin tanımlanması için de kullanışlıdır. Ölçüm sistemi, 0.1-20 MHz. uyartım frekans aralığına, $1 \mathrm{~Hz}$.'lik sinüs sinyal adım çözünürlüğüne, 0-10 V. 'luk uyartım gerilimine ve $10 \mathrm{~nm}$.'lik yer değiştirme hassasiyetini ölçebilecek kapasiteye sahiptir.

\section{Teşekkür}

Celal Bayar Üniversitesi Bilimsel Araştırma Projeleri Koordinasyon Birimine, 2015-127 Nolu bilimsel araştırma projemize yaptığı katkı için teşekkür ederiz.

\section{Referanslar}

[1] Ajitsaria, J; Choe, S.Y., Shen, D.; Kim, D.J. Modeling and analysis of a bimorph piezo electric cantilever beam for voltage generation. Smart Materials and Structures. 2007; 16 ,447-454.

[2] Dakua, I.; Afzulpurkar, N. Piezoelectric Energy Generation and Harvesting at the Nano-Scale: Materials and Devices. Nanomat and Nanotech 2013; 3, 21.
[3] Croft, D; Shed, G.; Devasia, S. Hysteresis, Creep and Vibration Compensation for Piezoactuators: Atomic Force Microscopy Application, Journal of Dynamic Systems, Measurement, and Control. 1999; 123(1), 35-43.

[4] Zou, Q.; Lenag, K.K.; Sadoun, E.; Reed, M.J.; Devasia S. Control Issues in High-Speed AFM for Biological Applications: Collagen Imaging Example, Asian Journal of Control, 2004; 6(2), 164-178.

[5] Stöppler, G.; Douglas, S. Adaptronic Gantry Machine Tool with Piezoelectric Actuator for Active Error Compensation of Structural Oscillations at the Tool Centre Point, Mechatronics, 2008; 18(8), 426-433.

[6] Yang, W.; Lee, S.Y.; You, B.J. A Piezoelectric Actuator with a Motion-Decoupling Amplifier for Optical Disk Drives, Smart Material Structure 2010; 19(6), 065027.1-065027.

[7] Wei, J.J.; Qiu, Z.C.; Han, J.; Wang, Y.C. Experimental Comparison Research on Active Vibration Control for Flexible Piezoelectric Manipulator Using Fuzzy Controller, Journal of Intelligent and Robotic Systems. 2010; 59(1), 31-56.

[8] Norman, P.; Bäckström, M.; Rantatalo, M.; Svoboda, A.; Kaplan, A. A sophisticated platform for characterization, monitoring and control of machining, Measurement Science and Technology. 2006; 17(4), 847-854.

[9] Wang, X.D.; Li, N.; Liu, M.W.; Wang, L.D. Dynamic characteristic testing for MEMS micro-devices with base excitation, Measurement Science and Technology. 2007; 18, 17401747.

[10] Zhen, S.; Chen, B.; Yuan, L.; Li, M.; Liang, J.; Yu, B. A novel interferometric vibration measurement sensor with quadrature detection based on $1 / 8$ wave plate, Optics \& Laser Technology. 2010; 42, 362-365.

[11] Matthew, S.A.; Hooker, W.W. National Aeronautics and Space Administration Langley Research Center, Characterization of Multilayer Piezoelectric Actuators for Use in Active Isolation Mounts, 1997.

[12] Paralı, L.; Pechousek, J.; Şabikoğlu, İ.; Novak, P.; Navarik, J.; Vujtek, M. A digital measurement system based on laser displacement sensor for piezoelectric ceramic discs vibration characterization, Optik - International Journal for Light and Electron Optics. 2016; 127, 84-89. 